\title{
Programmed cell death protein 1 on natural killer cells: fact or fiction?
}

\author{
Monica M. Cho, ${ }^{1}$ Aicha E. Quamine, ${ }^{1}$ Mallery R. Olsen, ${ }^{1}$ and Christian M. Capitini ${ }^{1,2}$ \\ 'Department of Pediatrics and ${ }^{2}$ Carbone Cancer Center, University of Wisconsin School of Medicine and Public Health, Madison, Wisconsin, USA.
}

\begin{abstract}
Programmed cell death protein 1 (PD-1) has become one of the most investigated targets for cancer immunotherapy. Most research has centered on inhibiting PD-1 on T cells, but there is increased interest in understanding the role of PD-1 on NK cells. While the expression of PD-1 on NK cells has been controversial, with papers publishing contradictory results in multiple models, there is increased clinical interest in NK and PD-1 immunotherapy. In this issue of the $J C I$, Judge et al. comprehensively explore the lack of $\mathrm{PD}-1$ expression on murine, canine, and human NK cells and the clinical implication of these findings.
\end{abstract}

\section{Checkpoint blockade}

Understanding the role of immune checkpoints has undeniably changed the landscape of cancer immunotherapy. Programmed cell death protein 1 (PD-1) is induced after $\mathrm{T}$ cells are activated and serves as a marker of activation that provides inhibitory signals to $\mathrm{T}$ cells after engagement of its ligand, programmed death ligand 1 (PD-L1). PD-1 is also a marker of chronic antigen stimulation, since checkpoint blockade with monoclonal antibodies against PD-1 abrogates $\mathrm{T}$ cell exhaustion, generating robust antitumor responses $(1,2)$. Cancers that have been clinically approved for PD-1 inhibition include melanoma, renal cell carcinoma, metastatic non-small cell lung cancer, urothelial carcinoma, classical Hodgkin lymphoma, and others $(3,4)$. Interestingly PD-1 blockade can induce responses in various cancers with low expression of major histocompatibility complex class I (MHCI), necessary for cytotoxic $\mathrm{CD} 8^{+} \mathrm{T}$ cell recognition, suggesting enhanced cytolytic activity by $\mathrm{CD} 4^{+} \mathrm{T}$ cells or effector cells capable of cytotoxicity independently of MHC.
NK cells are cytotoxic lymphocytes that can eliminate virally infected cells and tumors using cytotoxicity mechanisms similar to $\mathrm{CD} 8^{+} \mathrm{T}$ cells, but that do not require recognition of MHC (5). Using deductive reasoning from the "missing self" hypothesis, blocking PD-1 on NK cells may augment antitumor effects for tumors that are refractory to $\mathrm{T}$ cell treatments due to low MHC expression (6). The fundamental basis behind applying checkpoint blockade against PD-1 on NK cells must, of course, assume that NK cells express PD-1.

\section{PD-1 expression on activated murine NK cells}

There is no doubt that studies of PD-1 expression on NK cells, albeit with different methods of isolating and activating NK cells as well as of evaluating PD-1 expression, report conflicting findings. In this issue of the JCI, Judge et al. comprehensively examine induction of PD-1 expression by NK cells in multiple in vitro and in vivo models, providing direct comparisons among murine, canine, and human NK cells and validating PD-1 expression in tan-

Related Article: p. 3051

Authorship note: MMC, AEQ, and MRO contributed equally to this work.

Conflict of interest: CMC has a patent unrelated to this publication (US patent 10,166,254 B2).

Copyright: ( 2020, American Society for Clinical Investigation.

Reference information: / Clin Invest. 2020;130(6):2816-2819. https://doi.org/10.1172/JCI137051. dem with other activation and exhaustion markers using multiple techniques, including flow cytometry, quantitative real-time PCR (qRT-PCR), and RNA-sequencing (7). The authors first evaluated in vitro IL-2-activated murine adherent lymphokine-activated killer (ALAK) cells as well as purified murine NK cells by flow cytometry and RNA-sequencing and found minimal expression of PD-1. This PD- 1 analysis was repeated in $\mathrm{Rag}^{-1-}$ mice, who lack $\mathrm{B}$ and $\mathrm{T}$ cells but have NK cells, and diet-induced obese mice, a model known to promote $\mathrm{T}$ cell exhaustion and PD-1 expression (7). The researchers failed to detect PD-1 expression on NK cells in both of these models. Recent data had shown that intratumoral NK cells express PD-1 and mediate antitumor responses (8), but using multiple murine tumor cell lines implanted into syngeneic mice, the authors found few intratumoral NK cells and minimal PD-1 expression by flow cytometry and qRTPCR. A fluorescent reporter was used to measure intracellular or extracellular PD-1 expression in mice bearing CT26 colon cancer cells, but tumor-bearing mice failed to reveal PD-1 expression in NK cells, confirming that the results did not reflect an inability to detect PD-1 due to intracellular localization (7). Early response to CMV infection is known to result in marked NK activation (9), so a murine CMV (MCMV) infection model was used to look for induction of PD-1 expression on NK cells. While PD-1 expression remained stable after exposure to MCMV, TIGIT, another exhaustion marker, showed increased expression with MCMV infection in WT and $\mathrm{Rag}^{-1-}$ mice. Curiously, murine NK cells expressed minimal to no PD- 1 in these various inflammatory models, but perhaps human NK cells would be different (7).

\section{PD-1 expression on NK cells} after ex vivo activation

Next, Judge and authors evaluated PD-1 expression on human NK cells activated after in vitro cytokine stimulation with IL-2 


\section{Table 1. Summary of NK PD-1 expression studies}

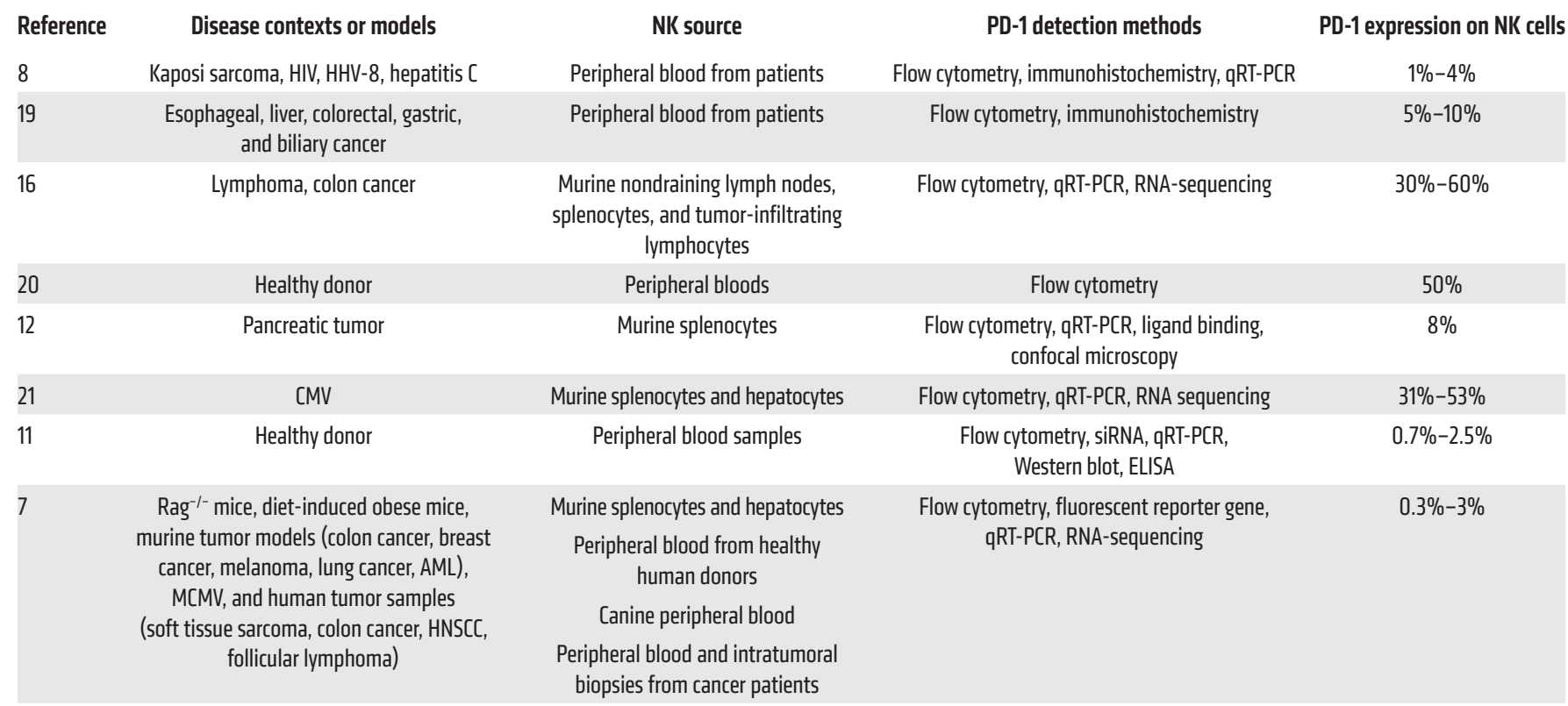

AML, acute myeloid leukemia; HHV-8, human herpesvirus-8; HNSCC, head and neck squamous cell carcinoma; MCMV, murine cytomegalovirus.

for 3 days or ex vivo expansion with IL-2 and feeder cells for 21 days. The researchers activated human peripheral blood mononuclear cells in vitro with concanavalin $\mathrm{A}$, a mitogen, and IL-2 or IL-15. This activation led to significantly increased PD-1 expression on T cells, but not NK cells, as detected by flow cytometry. Ex vivo expansion and activation of human NK cells via coculture with IL-2 and irradiated human K562 feeder cells expressing membrane bound IL-21 and CD137 ligand upregulated TIGIT, CD69, granzyme B and CD107a, demonstrating NK cell activation. However, the coculture did not result in increased PD-1 expression (by flow cytometry or qRT-PCR), even when IL-15 was added. Canine NK cells also cultured with the same feeder cells for 21 days did not exhibit increased PD-1 expression, as determined by qRT-PCR (7).

\section{PD-1 expression from human NK cells in cancer patients}

Human solid tumors can respond to NK cell therapy (10), so specimens from patients with solid tumors were used to evaluate PD-1 expression on peripheral blood and tumor-infiltrating NK cells. In the context of these tumors, peripheral and tumorinfiltrating NK cells were found to express the immune receptor TIGIT, but not PD-1, by flow cytometry. Even in murine and human hematologic malignancies, IL-2activated NK cells did not express PD-1. In summary, Judge et al. performed a thorough analysis for the expression of PD-1 on multiple species of NK cells using multiple readout methods and repeatedly demonstrated that NK cells, activated by various conditions, such as cytokine stimulation or feeder cells, or by contexts such as obesity, infection, or tumor, all result in minimal PD-1 expression. The results in this study conflict with PD-1 expression demonstrated using models from other studies (Table 1), but also highlight unique facets of the biology of PD-1 expression in NK cells and inconsistencies in how investigators measure PD-1 expression.

\section{Potential mechanisms driving false-positive PD-1 expression in NK cells}

RT-PCR and Western blot data have shown, using both human NK cell lines and purified NK cells from healthy donors, that resting NK cells and IL-2-stimulated NK cells harbor a cytoplasmic pool of PD-1 mRNA and protein despite low surface-expression levels (11). These data demonstrate that PD-1 may cycle to the NK cell surface for externalization, but requires appropriate stimuli (Figure 1).
Different NK cell subsets can produce splicing variants of PD-1 for which their potential effect on NK cell regulation cannot be overlooked (Figure 1). Differences in tissue processing between groups could result in PD-1 expression that is essentially a false positive (Figure 1). For instance, PD-1-specific antibodies can crossreact with a nuclear antigen that is released by dying cells upon nuclear envelope rupture (12). Judge and colleagues costained with a fixable viability dye to verify that PD-1 binding was confined to the dead cell despite discriminating for small size in the flow cytometry assay (7). These results suggest that studies need to account for quality control of their antibodies and/ or tissue processing while characterizing PD-1 expression and highlight how the methodology behind flow cytometry can influence cell metabolism, increasing the risk of cell death and thus the appearance of PD-1 expression.

Benson et al. was one of the initial groups to confirm PD-1 expression on isolated NK cells from patients with multiple myeloma (13). Although inactivated NK cells from healthy donors lacked PD-1, NK cell stimulation with $150 \mathrm{IU} / \mathrm{mL}$ IL-2 for 48 hours increased PD-1 expression from $1.4 \%$ to $16 \%$, as measured by flow cytometry. Differential doses of IL-2 


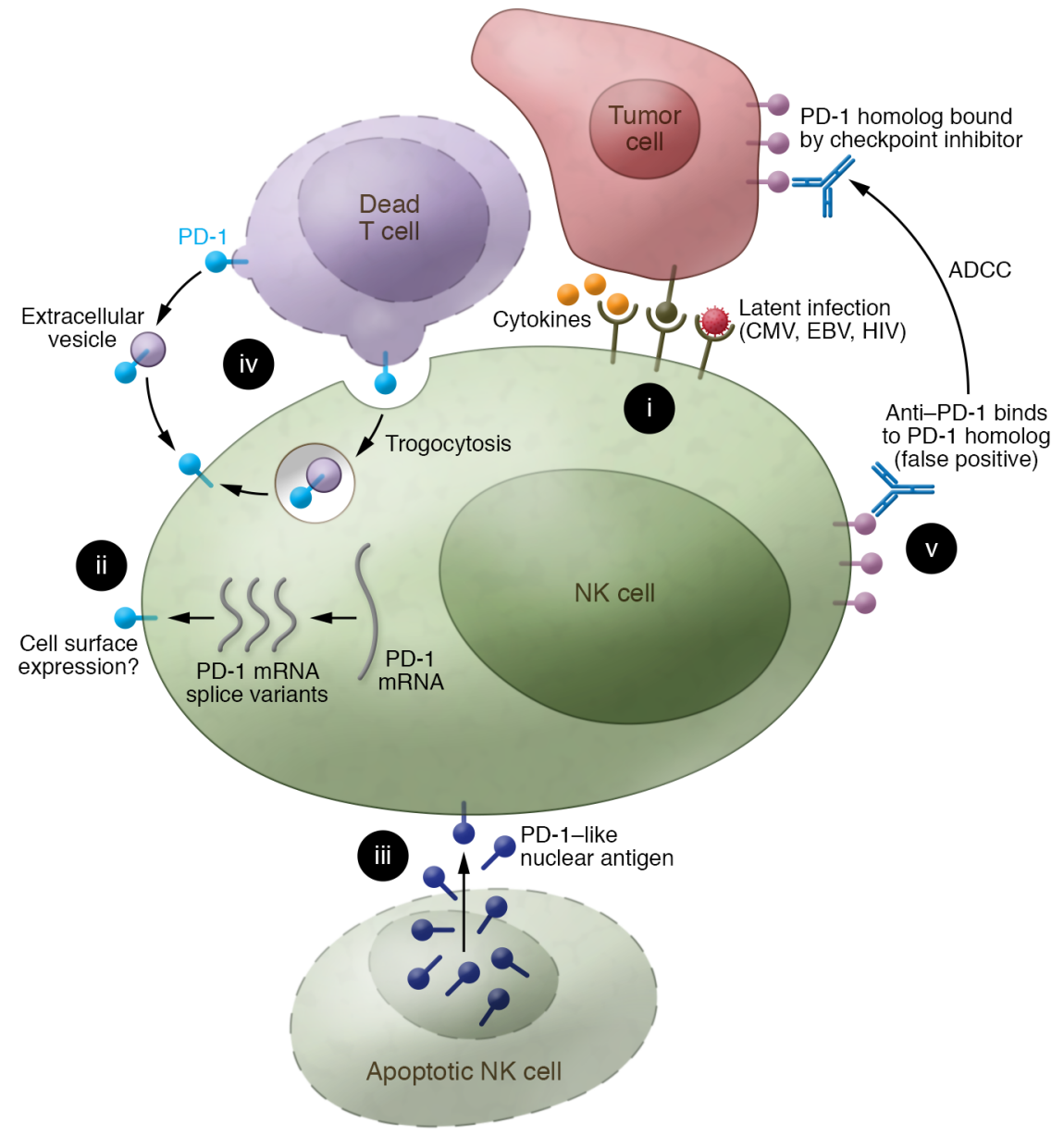

and incubation times are commonplace among different research groups (14-16), as exemplified in the current study (7), which used 1000 IU/mL IL-2 for 72 hours with human NK cells and for 7 days with murine NK cells. Perhaps overstimulating NK cells with cytokines can paradoxically suppress PD-1 expression or cause production of splice variants that fail to stimulate protein expression (Figure 1). While the Judge et al. results (7) show minimal PD-1 expression after MCMV infection, PD-1 expression has been identified in subsets of mature dysfunctional human NK cells in posttransplantation lymphoproliferative disorders (17) and after EBV, HIV, and human CMV infection $(6,18)$. While the human viral infection studies relied solely on flow cytometry to measure PD-1 expression, many studies included functional experiments with anti-PD-1 agents verifying enhanced NK cell activity. Thus PD-1 expression on NK cells may be species and infection specific as well as even patient dependent. Finally, in the context of cancer, it is possible that the antitumor benefits seen by others with anti-PD-1 therapy may have alternative explanations. Perhaps the tumor microenvironment provides complex immune cell interactions that cause dynamic changes in PD-1 expression that are difficult to recapitulate in vitro or identify in vivo with the limited murine NK cells that traffic to subcutaneous tumor models. One could imagine NK cells picking up PD-1 from other cells within the tumor, such as T cells, by trogocytosis or even through extracellular vesicles (Figure 1). Or perhaps the tumor has homologous receptors to $\mathrm{PD}-1$ that antibodies recognize yet that induce antibody-dependent cellular cytotoxicity via CD16 expression on NK cells (Figure 1).

\section{Conclusion}

As Judge et al. suggest, PD- 1 expression on murine, canine, and human NK cells remains minimal to absent, especially compared with expression on T cells (7). Consistency in NK cell activation methods
Figure 1. Potential mechanisms of PD-1 expression on NK cells and anti-PD-1 treatment. (i) When activating receptors on NK cells engage with ligands on tumor cells, cytokine receptors with cytokines or viral receptors with associated viruses. (ii) PD-1 mRNA and splice variants may (or may not) lead to PD-1 protein cell surface expression. (iii) NK cell apoptosis can result in release of a nuclear antigen that can be mistaken for PD-1. While NK cells may inherently minimally express PD-1, even after activation, NK cells could acquire PD-1 through (iv) uptake of extracellular vesicles released from T cells or by trogocytosis upon contact with a neighboring $T$ cell within the tumor microenvironment. (v) False-positive results could result from antiPD-1 labeling a currently unidentified cell surface homolog of PD-1 on NK cells. Benefits from anti-PD-1 checkpoint blockade could also result from anti-PD-1 binding a homolog on the tumo cell, which instead of preventing exhaustion in the NK cells, in fact, stimulates antibodydependent cellular cytotoxicity (ADCC). combined with rigorous cell surface and intracellular analysis of PD-1 expression may provide a more consistent understanding of the role, if any, of the PD-1/ PD-L1 pathway in NK cells. The clinical relevance of PD-1 checkpoint blockade on NK cells may become clearer as better detection methods and reagents are developed. More data, specifically from patients given anti-PD-1 who are deficient in T cells (such as in HIV or early after bone marrow transplant) or chimeric antigen receptormodified NK cells that are engineered as PD-1 deficient, will ultimately inform the debate on whether PD-1 expression on NK cells is fact or fiction.

\section{Acknowledgments}

This work was supported by grants from the National Institute of General Medical Sciences/NIH T32 GM008692 (to $\mathrm{MMC}$ ), a Bridge to the Doctorate fellowship and a SciMed GRS fellowship (to AEQ), the Ruth M. Vetter Fellowship in Pediatric Hematology, Oncology and 
Bone Marrow Transplant (to MRO), St. Baldrick's Stand up to Cancer (SU2C) Pediatric Dream Team Translational Research Grant SU2C-AACR-DT-27-17, NCI/NIH R01 CA215461, American Cancer Society Research Scholar Grant RSG18-104-01-LIB, and the Midwest Athletes Against Childhood Cancer (MACC) Fund (to CMC). SU2C is a division of the Entertainment Industry Foundation. Research grants are administered by the American Association for Cancer Research, the scientific partner of SU2C. The contents of this article do not necessarily reflect the views or policies of the Department of Health and Human Services, nor does mention of trade names, commercial products, or organizations imply endorsement by the US government.

Address correspondence to: Christian M. Capitini, University of Wisconsin, 1111 Highland Avenue, WIMR 4137, Madison, Wisconsin 53705, USA. Phone: 608.262.2415; Email:ccapitini@pediatrics.wisc.edu.

1. Chauvin JM, et al. TIGIT and PD-1 impair tumor antigen-specific $\mathrm{CD}^{+} \mathrm{T}$ cells in melanoma patients. J Clin Invest. 2015;125(5):2046-2058.

2. Fourcade J, et al. Upregulation of Tim-3 and PD-1 expression is associated with tumor antigen-specific CD8+ T cell dysfunction in melanoma patients. J Exp Med. 2010;207(10):2175-2186
3. Fessas P, Lee H, Ikemizu S, Janowitz T. A molecular and preclinical comparison of the PD-1-targeted T-cell checkpoint inhibitors nivolumab and pembrolizumab. Semin Oncol. 2017;44(2):136-140.

4. Juliá EP, et al. Peripheral changes in immune cell populations and soluble mediators after antiPD-1 therapy in non-small cell lung cancer and renal cell carcinoma patients. Cancer Immunol Immunother. 2019;68(10):1585-1596.

5. Concha-Benavente F, Kansy B, Moskovitz J, Moy J, Chandran U, Ferris RL. PD-L1 mediates dysfunction in activated PD- $1^{+}$NK cells in head and neck cancer patients. Cancer Immunol Res. 2018;6(12):1548-1560.

6. Kärre K, Ljunggren HG, Piontek G, Kiessling R. Selective rejection of $\mathrm{H}$-2-deficient lymphoma variants suggests alternative immune defence strategy. Nature. 1986;319(6055):675-678.

7. Judge SJ, et al. Minimal PD-1 expression in mouse and human NK cells under diverse conditions. J Clin Invest. 2020;130(6):3051-3068.

8. Beldi-Ferchiou A, et al. PD-1 mediates functional exhaustion of activated NK cells in patients with Kaposi sarcoma. Oncotarget. 2016;7(45):72961-72977.

9. Arase H, Lanier LL. Virus-driven evolution of natural killer cell receptors. Microbes Infect. 2002;4(15):1505-1512.

10. Cho D, Shook DR, Shimasaki N, Chang YH, Fujisaki H, Campana D. Cytotoxicity of activated natural killer cells against pediatric solid tumors. Clin Cancer Res. 2010;16(15):3901-3909.

11. Mariotti FR, et al. PD-1 in human NK cells: evidence of cytoplasmic mRNA and protein expression. Oncoimmunology. 2019;8(3):1557030.

12. Metzger $P$, et al. Dying cells expose a nuclear antigen cross-reacting with anti-PD-1 monoclonal antibodies. Sci Rep. 2018;8(1):8810.
13. Benson DM, et al. The PD-1/PD-L1 axis modulates the natural killer cell versus multiple myeloma effect: a therapeutic target for CT-011, a novel monoclonal anti-PD-1 antibody. Blood. 2010;116(13):2286-2294.

14. Chen J, et al. Programmed cell death protein-1/ programmed death-ligand 1 blockade enhances the antitumor efficacy of adoptive cell therapy against non-small cell lung cancer. J Thorac Dis. 2018;10(12):6711-6721.

15. Pesce S, et al. PD/1-PD-Ls checkpoint: insight on the potential role of NK cells. Front Immunol. 2019;10:1242.

16. Hsu J, et al. Contribution of NK cells to immunotherapy mediated by PD-1/PD-L1 blockade. J Clin Invest. 2018;128(10):4654-4668.

17. Wiesmayr S, et al. Decreased NKp46 and NKG2D and elevated PD-1 are associated with altered NK-cell function in pediatric transplant patients with PTLD. Eur J Immunol. 2012;42(2):541-550.

18. Wenjin Z, Chuanhui P, Yunle W, Lateef SA, Shusen Z. Longitudinal fluctuations in PD1 and PD-L1 expression in association with changes in anti-viral immune response in chronic hepatitis B. BMC Gastroenterol. 2012;12:109.

19. Liu Y, et al. Increased expression of programmed cell death protein 1 on NK cells inhibits NK-cell-mediated anti-tumor function and indicates poor prognosis in digestive cancers. Oncogene. 2017;36(44):6143-6153.

20. Lieberman NAP, et al. An uncoupling of canonical phenotypic markers and functional potency of ex vivo-expanded natural killer cells. Front Immunol. 2018;9:150.

21. Quatrini L, et al. Endogenous glucocorticoids control host resistance to viral infection through the tissue-specific regulation of PD-1 expression on NK cells. Nat Immunol. 2018;19(9):954-962. 